DOI: 10.4274/ejgg.galenos.2019.216

Eur J Geriatr Gerontol 2020;2(1):24-25

\title{
Delusion of Pregnancy in a Case of Dementia
}

\author{
(D) N.A. Uvais \\ Iqraa International Hospital and Research Centre, Clinic of Psychiatry, Calicut, India
}

\begin{abstract}
Delusion of pregnancy is a disorder characterised by a false and fixed belief of being pregnant despite factual evidence to the contrary. Although delusions are a common and troublesome feature of dementia, delusions of pregnancy have rarely been reported. We report the case of a 70-yearold widowed female with dementia who presented with delusion of pregnancy. She was not taking any antipsychotic medications prior to the onset of delusion and her serum prolactine level was normal.
\end{abstract}

Keywords: Delusion of pregnancy, dementia, elderly

\section{Introduction}

Delusion of pregnancy is a disorder characterised by a false and fixed belief of being pregnant despite factual evidence to the contrary. This psychopathological symptoms has been reported in women of all age group as well as in men. A recent systematic review of 84 cases of delusion of pregnancy published in the literature showed that this condition predominantly affect females between 20-40 years of age and often seen associated with schizophrenia, bipolar disorders and psychotic depression (1). There are also rare reports of delusion of pregnancy secondary to various organic disorders such as epilepsy, motor neurone disease, delirium, Metabolic syndrome, primary heperprolactinaemia, hashimoto thyroidits, sarcoidosis, primary sterility due to tubal cyst, hypothyroidism and intermittent polydipsia and Hyponatremia syndrome (1). There are also occasional reports of delusion of pregnancy in patients with dementia. Here, we report the case of a 70-years-old female with dementia who presented with delusion of pregnancy.

\section{Case Report}

A 76 year-old childless widow was brought to the psychiatry outpatient department by her relatives with a history of progressive memory loss over the last six years. She had become increasingly disinhibited and restless over the previous 12 months. She also started to state repeatedly that she was pregnant. She often enquire the safety of the medications during pregnancy before taking it and resist activities where she need to bow down. There was no history of weight gain or constipation. There was no past history of medical or psychiatric illness. There was no significant family history of any medical or psychiatric illness. Abdominal examination was normal.

During mental status examination she reported that she is six month pregnant. There was no hallucinations or other delusions. Her affect was cheerful. She showed significant impairment in both recent and remote memory. She scored 19 in mini-mental state examination. Structural neuroimaging showed age related brain atrophy. Serum prolactin level was with in normal limits. Using the Cambridge behavioural inventory, a checklist of cognitive and behavioural symptoms, which was completed by the patient's brother, there was also evidence for impaired memory and orientation, loss everyday skills, impaired self care, mood fluctuations, abnormal behaviour, beliefs in odd or bizarre ideas that cannot be true, poor table manners, disturbed sleep and indifferent to the worries of family members. She was diagnosed with Alzheimer's dementia with behavioural and psychological symptoms.

\section{Discussion}

There are few published reports of delusion of pregnancy in patients with Alzheimer's dementia. Guilfoyle et al. (2) reported

Address for Correspondence: N.A. Uvais, Iqraa International Hospital and Research Centre, Clinic of Psychiatry, Calicut, India Phone: +09562685573 E-mail: druvaisna@gmail.com ORCID: orcid.org/0000-0003-0961-3025

Received: Oct 18, 2019 Accepted: Nov 18, 2019

Cite this article as: N.A. Uvais. Delusion of Pregnancy in Case of Dementia. Eur J Geriatr Gerontol 2020;2(1):24-25

๑Copyright 2020 by the Academic Geriatrics Society / European Journal of Geriatrics and Gerontology published by Galenos Publishing House. 
a case series of five elderly woman aged between 74-89 years. Out of the five patients, two had a dementing illness. Cipriani \& Fiorino done a systematic literature review to identify the characteristic of a psychotic symptom, the phenomenon of delusion of pregnancy, in the context of dementia. They described three case reports of delusion of pregnancy in Alzhiemer's dementia and two case reports in frontotemporal dementia (3). Curran and Pugh described three elderly patients with delusion of pregnancy, of which two had dementia (4).

Various psychological and socio-cultural factors have been implicated in the causation of delusion of pregnancy. Even though delusion of pregnancy occurred in our patient in the setting of a dementing illness, it appeared to indicate wish fulfilment. She had a repressed sexual life and had no children.

Treatment with anti-psychotic medications is an important cause of delusion of pregancy since its use are associated with hyperprolactinaemia and weight gain. However, our patient was not on any anti-psychotics prior to the onset of the delusion of pregnancy and her serum prolactine level was normal. Most of the past reports also described distended abdomens and constipation associated with delusion of pregnancy. However, our patient did not show these symptoms.

\section{Ethics}

Informed Consent: Informed consent was obtained from the patients or their relatives.

Peer-review: Internally peer-reviewed.

Financial Disclosure: The authors declared that this study received no financial support.

\section{References}

1. Bera SC, Sarkar S. Delusion of pregnancy: a systematic review of 84 cases in the literature. Indian J Psychol Med 2015;37:131-137.

2. Guilfoyle $P, O^{\prime} B r i e n ~ H, ~ O ' K e e f f e ~ S T$. Delusions of pregnancy in older women: a case series. Age and Ageing 2015;44:1058-1061.

3. Cipriani G, Di Fiorino M. Delusion of Pregnancy: an unusual symptom in the context of dementia. American Journal of Alzheimer's Disease Other Dementias 2014;30:341-345.

4. Curran JSM, Pugh RE. Delusions of pregnancy and delivery in elderly women. International Journal of Geriatric Psychiatry 1995;10:1075-1076. 